\title{
Choice of the RF Cavity for the SSC Collider
}

\author{
W. Chou \\ Superconducting Super Collider Laboratory* \\ 2550 Beckleymeade Ave., Dallas, TX 75237
}

\begin{abstract}
Four types of RF cavities - multiple cell and single cell, superconducting (sc) and normal conducting (nc) - have been compared with respect to the specific needs of the SSC Collider. The single cell cavity is preferable to the multi-cell one because its higher order modes (HOM) are easier to damp. The sc cavity has a number of advantages over the nc one. But its mechanical complexity and operational reliability may present a concern. The impact of different if frequencies on the beam parameters have also been studied. In the range from $180 \mathrm{MHz}$ to $480 \mathrm{MHz}$, the parameter variations are generally small.
\end{abstract}

\section{INTRODUCTION}

In the SSC Collider, the beam intensity of a single bunch is limited by the detectors. Therefore, in order to achieve the design luminosity $10^{33} \mathrm{~cm}^{-2} \mathrm{~s}^{-1}$, one has to use about 17,000 bunches in each ring. The coupled bunch instability is a main concern. The use of a wideband feedback system to damp the instability is difficult because it can lead to emittance dilution, which has been observed in the Tevatron and SPS. An effeclive way to avoid the instability is to employ passive damping, i.e., to damp the HOM such that all the unstable bunch modes would be Landau damped.

The Collider if parameters are: $f_{\mathrm{rf}}=360 \mathrm{MHz}, V_{\mathrm{rf}}=20$ $\mathrm{MV}$, and $P_{\mathrm{rf}}=2 \mathrm{MW}$. The frequency must be a multiple of $60 \mathrm{MHz}$, which is the basic bunch spacing. The voltage is mainly determined by the requircment of the bucket size (in order to avoid the rf noise problem while accommodating a beam with large longitudinal emittance) rather than by the acceleration (which needs $3.9 \mathrm{MV} /$ turn during ramping). The power requirement comes from the beam loading compensation during storage (1.4 MVA, reactive), with adequate safety margin.

The baseline design is to use eight 5-cell cavities of the PEP type and two $1 \mathrm{MW}$ klystrons [1]. However, the calculations show that the HOM of this cavity would cause longitudinal and transverse multibunch instabilities at injection $(2 \mathrm{TeV})$. The e-folding time is in the order of seconds. At top energy $(20 \mathrm{TeV})$, the unstable modes would be Landau damped, but only marginally. Therefore, there was a proposal to change the baseline by adopting the single cell type cavity [2].

* Operated by the Universities Research Association, Inc., fur the U.S. Department of Energy under Contract No. DE-AC3589ER40486.

\section{CRITERIA OF CHOICE}

\section{A. HOM and coupled bunch instability}

For the rf cavity in the Collider, HOM damping is a major design goal. If the HOM are not properly damped, the coupled bunch instability will occur, as experienced in the LEP and HERA. When a wideband feedback system is employed, the noises in the feedback system may blow up the beam emittance. The required transverse emittance ( $1 \mathrm{~mm}-\mathrm{mrad}$, rms, normalized) of the Collider beam is demanding. Any increase in emittance means the decrease in luminosity. The emittance growth rate due to the feedback noise is [3]:

$$
\frac{1}{\tau} \equiv \frac{\dot{\epsilon}}{\epsilon}=0.64 f_{0}\left(\frac{x_{N}}{\sigma_{x}}\right)^{2} \Delta \nu^{2}
$$

in which $f_{0}$ is the revolution frequency, $\sigma_{x}$ the rms beam width, and $\Delta \nu$ the betatron tune spread. The pickup resolution of the feedback system, $x_{N}$, is proportional to $\Delta f^{1 / 2}$, where $\Delta f$ is the bandwidth of the feedback system. A wider bandwidth gives rise to a larger thermal noise. Therefore, it is preferred that the HOM of the cavity can be damped such that the wide-band feedback system will become unnecessary.

\section{B. Transient beam loading}

In the train of the Collider bunches, there are seven injection gaps $(1.7 \mu \mathrm{s})$ and one abort gap $(4.2 \mu \mathrm{s})$. These gaps will cause phase modulation. In the linear approximation, the modulation amplitude is:

$$
\Delta \phi=\left(\frac{I_{\mathrm{b}} R}{V_{\mathrm{c}}}\right) \cdot\left(\frac{\omega_{\mathrm{rf}}}{2 Q}\right) \Delta t
$$

in which $R, Q$ and $\omega_{\mathrm{rf}}$ are the shunt impedance, quality factor and angular frequency of the cavity, respectively, $V_{c}$ the cavity voltage, $I_{\mathrm{b}}$ the rf component of the beam current $\left(I_{\mathrm{b}}=2 I_{\mathrm{dc}}\right)$, and $\Delta t$ the gap size. When the ring is partially filled (for instance, during the injection), this formula is no longer valid and the computation is more involved. The consequences of the phase modulation are: (a) The jiggering of the interaction point in the longitudinal direction. (b) The reduction of the luminosity due to a crossing angle. (c) A larger occupation of the bucket by the oscillating bunch. Therefore, $\Delta \phi$ has to be limited under certain value by feedforward or fast feedback. 


\section{Detuning and fundamental mode instability}

The optimum detuning for reactive compensation is:

$$
\frac{\Delta f_{\mathrm{rf}}}{f_{\mathrm{o}}}=\frac{I_{\mathrm{b}} \cos \phi_{\mathrm{b}}}{2 V_{\mathrm{c}}} \cdot \frac{R}{Q} \cdot h
$$

where $h$ is the harmonic number. For a fully detuned nc cavity in the Collider, $\Delta f_{\mathrm{rf}}$ is about $-6 \mathrm{kHz}$. Because $f_{0}$ is low $(3.441 \mathrm{kHz})$, the impedance of the fundamental mode will cover several revolution lines. Thus, detuning will strongly drive the following coupled bunch modes unstable: $m=1, n=-1,-2,-3, \ldots$ If, on the other hand, not to detune the cavity (at a price of higher power losses) in order to avoid this problem, then the operation and control of the cavity may become difficult. A possible solution is to employ a fast $\mathbf{r f}$ feedback, which is widely used at CERN. In this case, the total loop delay is an utmost important parameter and should be made as short as possible when planning the klystron gallery location.

\section{Broadband impedance}

In addition to the HOM, the rf cavities also have a broadband impedance, which will affect the single bunch instabilities. However, in the Collider impedance budget, the contribution from the of cavities is small ( $10 \%$ in longitudinal and $0.1 \%$ in transverse). Therefore, this should not be a big issue in making the choice of the cavity.

\section{E. RF noise}

The phase and amplitude rf noises can cause longitudinal emittance growth, particle loss and reduction of the beam lifetime. Thanks to a large bucket-to-bunch-area ratio in the design ( 30 at injection and 25 at full energy), this is not a serious problem. Study shows that the emittance doubling time exceeds 50 hours at the specified noise level [4]. One special feature of the Collider is that the synchrotron frequency is so low $(4 \mathrm{~Hz})$ that the sideband will always fall into the impedance bandwidth of the cavity no matter it is nc or sc. (This is in contrast to the LEP, in which the synchrotron frequency of the proton beam is several hundreds hertz. Improvement of beam lifetime was observed when sc cavities were used there.)

\section{F. Reliability and availability}

Because of the big size of the SSC, the reliability and availability of the of cavity is a top priority issue. This includes: (a) The failure probability of the cavity; (b) When a cavity is tripped, whether the beam can survivc; (c) If the beam has to be dumped, whether an injection is immediately possible. In this regard, it is obvious that using two large klystrons is not a good design. Because the failure of one cavity can lead to the loss of half rf voltage. Using four or eight klystrons is a better scheme. The $500 \mathrm{~kW}$ and 250 $\mathrm{kW}$ klystron can be available on the market (Varian and Litton) if one needs them. The trip rate of the cavity will be discussed later.

\section{G. Flexibility}

It is desirable to use the cavity as a kicker to damp the injection errors and low order coupled bunch instability. For this purpose, the klystron should have enough reserved power and the system should have enough bandwidth (about $500 \mathrm{kHz}$ ), which can be achieved by using an rf feedback.

\section{H. Power limitation of the window}

The power of each window should not exceed $200 \mathrm{~kW}$ by today's technology. Otherwise the manufacture will be difficult and the reliability will be questionable.

\section{RF power loss}

This is always an important issue in electron machines (e.g., the B-factory). But it is much less so in the SSC, which is a proton machine. The reactive power of the rf cavities is 1.4 MVA, which is a small fraction of the total installed power (about 0.5 GVA). Either detuning or not detuning, sc or nc, this issue does not play a big role.

\section{COMPARISONS}

\section{A. Multi-cell, nc cavity}

This type is cheaper than the single cell cavity. But the problem is that its HOM are not easy to damp because of lack of room for the HOM couplers and because of the cross-talk between neighboring cells. When DORIS installed the PETRA 5-cell cavity, it tried to damp the HOM by inserting couplers through the pickup flanges. The impedance reduction was limited by a factor of $2-3$ [5]. A more successful case was the CESR 14-cell cavity, in which the HOM have been damped by a factor of 20-100 [6]. Another concern is the window power that will exceed $200 \mathrm{~kW}$. The two-window-per-cavity design requires a significant amount of $R \& D$ work.

\section{B. Multi-cell, sc cavity}

This type is excluded because of the following reasons: (a) If two 5-cell sc cavities are used, each window would take $1 \mathrm{MW}$, which is far beyond the allowable maximum of $200 \mathrm{~kW}$. (b) The HOM couplers can only be installed near the ends of the multi-cell structure in order to avoid multipacting. This makes it difficult to damp those HOM that are confined in the middle part of the cavity.

\section{Single cell, nc cavity}

This type is ideal for HOM daming, which can be achieved by either installing couplers and/or by staggered tuning. A recent example is the cavity being developed at the Advanced Light Source at ANL. The damping factor is between 10 to 100 . 'The cavity suggested by SLAC/LBL for the B-factory has three waveguide attachments and looks promising. But considerable engineering efforts are needed before it can be put to use. The number of cavities is determined by the power dissipation in the wall (maximum $10 \mathrm{~W} / \mathrm{cm}^{2}$ ). It is possible to achieve $20 \mathrm{MV}$ by using 32 or even 24 cavities. 


\begin{tabular}{|c|c|c|c|c|c|}
\hline \multirow[t]{2}{*}{ Parameter } & \multicolumn{3}{|c|}{ RF Frequency } & \multirow{2}{*}{$\begin{array}{c}\text { Variation }^{\dagger} \\
\%\end{array}$} & \multirow[t]{2}{*}{ Note } \\
\hline & $480 \mathrm{MHz}$ & $360 \mathrm{MHz}$ & $180 \mathrm{MHz}$ & & \\
\hline Longitudinal emittance, $\mathrm{rms}(\pi \mathrm{eV}-\mathrm{s})$ & \multirow{2}{*}{\multicolumn{3}{|c|}{$\begin{array}{l}0.035 \\
0.233\end{array}$}} & - & inj, fixed \\
\hline & & & & - & $20 \mathrm{TeV}$, fixed \\
\hline RF voltage (MV) & \multicolumn{3}{|c|}{6.6} & - & inj, fixed \\
\hline & \multicolumn{3}{|c|}{20} & - & $20 \mathrm{TeV}$, fixed \\
\hline \multirow[t]{2}{*}{ Bunch length, rms (cm) } & 5.1 & 5.4 & 6.5 & $-6 /+20$ & inj \\
\hline & 5.6 & 6.0 & 7.1 & $-7 /+18$ & $20 \mathrm{TeV}$ \\
\hline \multirow[t]{2}{*}{ Energy spread, rms $\left(\times 10^{-5}\right)$} & 10.4 & 9.7 & 8.1 & $+7 /-16$ & inj \\
\hline & 6.3 & 5.8 & 4.9 & $+9 /-16$ & $20 \mathrm{TeV}$ \\
\hline \multirow[t]{2}{*}{ Synchrotron tune } & 0.0026 & 0.0022 & 0.0016 & $+18 /-27$ & inj \\
\hline & 0.0014 & 0.0012 & 0.0009 & $+17 /-25$ & $20 \mathrm{TeV}$ \\
\hline \multirow[t]{2}{*}{ Bucket area (eV-s) } & 2.17 & 3.34 & 9.44 & $-35 /+180$ & inj \\
\hline & 11.9 & 18.3 & 51.9 & $-35 /+180$ & $20 \mathrm{TeV}$ \\
\hline \multirow[t]{2}{*}{ Bucket-to-bunch-area ratio } & 20 & 30 & 86 & $-35 /+180$ & inj \\
\hline & 16 & 25 & 71 & $-35 /+180$ & $20 \mathrm{TeV}$ \\
\hline Luminosity reduction & 0.87 & 0.85 & 0.81 & $+2 /-5$ & $20 \mathrm{TeV}$ \\
\hline Particles per bunch $\left(\times 10^{10}\right)$ & 0.82 & 0.84 & 0.91 & $-2 /+8$ & $\operatorname{inj} / 20 \mathrm{TeV}$ \\
\hline Synchrotron radiation ( $\mathrm{kW} /$ beam) & 8.9 & 9.0 & 9.2 & $-1 /+2$ & $20 \mathrm{TeV}$ \\
\hline Parasitic heating ( $\mathrm{kW} /$ beam) & 0.89 & 0.73 & 0.50 & $+22 /-32$ & $20 \mathrm{TeV}$ \\
\hline Dynamic aperture $(\sigma)$ & 12.8 & 12.9 & 13.3 & $-1 /+3$ & inj \\
\hline Longi threshold impedance $(\Omega)$ & 3.9 & 3.6 & 2.8 & $+3 /-22$ & inj \\
\hline Trans threshold impedance ( $\mathrm{M} \Omega / \mathrm{m})$ & 260 & 238 & 185 & $+9 /-22$ & inj \\
\hline Longi intrabeam scattering (h) & 200 & 203 & 205 & $-1 /+1$ & $20 \mathrm{TeV}$ \\
\hline Trans intrabeam scattering (h) & 131 & 116 & 83 & $+13 /-28$ & $20 \mathrm{TeV}$ \\
\hline Space charge tune shift in LEB & 0.39 & 0.40 & 0.43 & $-3 /+8$ & inj \\
\hline
\end{tabular}

t The values at $360 \mathrm{MHz}$ are used as the reference.

\section{Single cell, sc cavity}

This type has a number of advantages: low rf loss, low broadband impedance, low transient beam loading (because of its low $R / Q$ and high $V_{c}$ ) and, most importantly, low HOM (because the total number of sc cavities is smaller than the nc cavities). But the main concern is the complexity of its structure and the associated reliability problem. There are reports about the high average trip rate of the sc cavities at TRISTAN (more than one trip per fill) [7] and CEBAF (one trip per cavity every 16 hours) [8]. The causes have not yet been fully understood. A more careful study is needed to assess the risks if sc cavities are to be used in the SSC.

\section{RF FREQUENCY}

When the longitudinal emittance and if voltage are fixed, many beam parameters have weak dependence on $f_{\mathrm{rf}}$, such as the rms bunch length $\left(\propto f_{\mathrm{rf}}^{-1 / 4}\right)$, rms energy spread ( $\left.\propto f_{\mathrm{rf}}^{1 / 4}\right)$, and synchrotron tune $\left(\propto f_{\mathrm{rf}}^{1 / 2}\right)$. The bucket size $\left(\propto f_{\mathrm{rf}}^{-3 / 2}\right)$ has a relatively strong dependence on $f_{\mathrm{rf}}$. The above table lists the beam parameters that correspond to three different $\mathrm{rf}$ frequencies. The variations of most parameters are small except the bucket area and bucket-to-bunch-area ratio, of which the changes are noticeable. This ratio determines the tolerable if noise level. Should this become an issue, one can enlarge the bucket by increasing the rf voltage $\left(\propto V_{\mathrm{rf}}^{1 / 2}\right)$. Other factors one needs to consider include:

- Beam transfer from the HEB to the Collider: The $\mathrm{HEB}$ rf is $60 \mathrm{MHz}$. One will have to rotate the beam in the longitudinal phase space before extracting it from the HEB. Higher of frequency in the Collider makes the injection more difficult.

- Cost: High frequency klystrons are cheaper.

- Market availability: There are $350 \mathrm{MHz}$ and $500 \mathrm{M} \mathrm{Hz}$ cavities available, which can be modified to $360 \mathrm{MHz}$ and $480 \mathrm{MHz}$, resp. Also available are the high power klystrons in the range $350-500 \mathrm{MHz}$.

\section{REFERENCES}

[1] Site Specific Conceptual Design, SSCL-SR-1056, (July 1990).

[2] W. Chou and G. Schaffer, "Comments on the Collider rf Cavity in the SSC," SSCL internal technical note PMTN-036C (January 1992).

[3] W. Chou and J. Peterson, SSCL-623 (1993).

[4] H.-J. Shih et al., SSCL-520 (1992).

[5] B. Dwersteg et al., IEEE Trans. Nucl. Sci. NS-32, p. 2797 (1985).

[6] R. Sundelin et al., IEEE Trans. Nucl. Sci. NS-28, p. 2844 (1981).

[7] K. Akai et al., KEK Preprint 91-35 (May 1991).

[8] R. Sundelin, private communication. 